\title{
New Constraints on Tau-Coupled Heavy Neutral Leptons with Masses $m_{N}=280-970 \mathrm{MeV}$
}

\author{
R. Acciarri, ${ }^{1}$ C. Adams, ${ }^{2}$ J. Asaadi, ${ }^{3}$ B. Baller,${ }^{1}$ V. Basque, ${ }^{1}$ F. Cavanna,${ }^{1}$ A. de Gouvêa, ${ }^{4}$ R. S. Fitzpatrick,${ }^{5}$ \\ B. Fleming, ${ }^{6}$ P. Green $\odot,{ }^{7, *}$ C. James, ${ }^{1}$ K. J. Kelly, ${ }^{1}$ I. Lepetic, ${ }^{8}$ X. Luo, ${ }^{9}$ O. Palamara, ${ }^{1}$ \\ G. Scanavini, ${ }^{6}$ M. Soderberg, ${ }^{10}$ J. Spitz, ${ }^{5}$ A. M. Szelc, ${ }^{11}$ W. Wu, ${ }^{1}$ and T. Yang ${ }^{1}$
}

(ArgoNeuT Collaboration)

${ }^{1}$ Fermi National Accelerator Lab, Batavia, Illinois 60510, USA
${ }^{2}$ Argonne National Lab, Lemont, Illinois 60439, USA
${ }^{3}$ University of Texas at Arlington, Arlington, Texas 76019, USA
${ }^{4}$ Northwestern University, Evanston, Illinois 60208, USA
${ }^{5}$ University of Michigan, Ann Arbor, Michigan 48109, USA
${ }^{6}$ Yale University, New Haven, Connecticut 06520, USA
${ }^{7}$ University of Manchester, Manchester M13 9PL, United Kingdom
${ }^{8}$ Rutgers University, Piscataway New Jersey 08854, USA
${ }^{9}$ University of California, Santa Barbara, California, 93106, USA
${ }^{10}$ Syracuse University, Syracuse, New York 13244, USA
${ }^{11}$ University of Edinburgh, Edinburgh EH9 3FD, United Kingdom

(Received 28 June 2021; accepted 12 August 2021; published 15 September 2021)

\begin{abstract}
A search for heavy neutral leptons has been performed with the ArgoNeuT detector exposed to the NuMI neutrino beam at Fermilab. We search for the decay signature $N \rightarrow \nu \mu^{+} \mu^{-}$, considering decays occurring both inside ArgoNeuT and in the upstream cavern. In the data, corresponding to an exposure to $1.25 \times 10^{20}$ POT, zero passing events are observed consistent with the expected background. This measurement leads to a new constraint at $90 \%$ confidence level on the mixing angle $\left|U_{\tau N}\right|^{2}$ of tau-coupled Dirac heavy neutral leptons with masses $m_{N}=280-970 \mathrm{MeV}$, assuming $\left|U_{e N}\right|^{2}=\left|U_{\mu N}\right|^{2}=0$.
\end{abstract}

DOI: 10.1103/PhysRevLett.127.121801

Introduction.-The discovery that neutrinos oscillate and therefore have mass has inspired numerous experimental efforts to understand this phenomenon. The standard model (SM) does not predict the existence of neutrino masses, requiring additional fields and/or interactions to generate them. One such model requires the existence of two or more heavy neutral leptons (HNLs): SM gauge singlet fermions that mix with the light neutrinos. This mixing can induce the observed small neutrino masses via one of many different seesaw mechanisms [1-7]. In addition, HNLs can provide solutions to other mysteries of nature such as the baryon asymmetry of the universe [8] (via leptogenesis) or dark matter [9]. In this Letter, we present a search for HNLs with masses $\mathcal{O}(100) \mathrm{MeV}$ using the ArgoNeuT detector.

We consider the simplest phenomenological scenario including a HNL, $N$ - that it has a mass $m_{N}$ and mixes with the light neutrinos via one or more nonzero new angles

Published by the American Physical Society under the terms of the Creative Commons Attribution 4.0 International license. Further distribution of this work must maintain attribution to the author(s) and the published article's title, journal citation, and DOI. Funded by SCOAP ${ }^{3}$.
$\left|U_{e N}\right|^{2},\left|U_{\mu N}\right|^{2}$, and $\left|U_{\tau N}\right|^{2}$ in an extended $4 \times 4$ leptonic mixing matrix. If $m_{N}$ is in the $\sim \mathrm{MeV}-\mathrm{GeV}$ range, HNLs can be produced as a result of high-energy proton-fixedtarget collisions, travel to a downstream detector and decay producing detectable charged particles. In the ArgoNeuT detector, we search for the decay signature $N \rightarrow \nu \mu^{+} \mu^{-}$.

ArgoNeuT was a 0.24 ton Liquid Argon Time Projection Chamber (LArTPC) neutrino detector located in the NuMI beam [10] at Fermilab that collected data in 2009-2010. The instrumented volume of the TPC was $40 \times 47 \times$ $90 \mathrm{~cm}^{3}$ (vertical, drift, beam direction) with two readout planes, each consisting of 240 wires spaced by $4 \mathrm{~mm}$ and oriented at $\pm 60^{\circ}$ to the horizontal. A detailed description of the design and operation of the ArgoNeuT detector can be found in Ref. [11]. The ArgoNeuT detector was located $100 \mathrm{~m}$ underground in the MINOS near detector hall, $1033 \mathrm{~m}$ downstream of the NuMI target and immediately upstream of the MINOS near detector (MINOS-ND). ArgoNeuT was able to use the MINOS-ND as a muon spectrometer. A detailed description of the MINOS-ND can be found in Ref. [12]. The analysis reported in this Letter is performed using $1.25 \times 10^{20}$ protons-on-target (POT) collected in reverse horn current (anti-neutrino) mode, during 
which both ArgoNeuT and the MINOS-ND were operational [11].

Generation and simulation.-A HNL with mass $m_{N}$ and mixing angle $\left|U_{\alpha N}\right|^{2}$ with the light neutrinos, $\nu_{\alpha}(\alpha=e, \mu$, $\tau)$, can be produced by any kinematically accessible process that would normally result in an outgoing $\nu_{\alpha}$. For the decay $N \rightarrow \nu \mu^{+} \mu^{-}$we require $m_{N}>2 m_{\mu}$ and, for simplicity, we assume that only one angle $\left|U_{\alpha N}\right|^{2}$ is nonzero at a time. A variety of experiments [13-21] have set powerful constraints on the angles $\left|U_{e N}\right|^{2}$ and $\left|U_{\mu N}\right|^{2}$ in the region of interest for ArgoNeuT. We therefore focus on the case where $\left|U_{\tau N}\right|^{2}$ is the only nonzero mixing angle. In this scenario, the HNLs are predominantly produced in the decays of $\tau^{ \pm}$leptons originating from decays of $D_{(s)}^{ \pm}$ mesons. The lifetime of $N$, as well as the branching ratio $\operatorname{Br}\left(N \rightarrow \nu \mu^{+} \mu^{-}\right)$, can be calculated as a function of the mixing $\left|U_{\tau N}\right|^{2}$ considering all kinematically accessible final states [22]. We assume that $N$ is a Dirac fermion throughout this analysis.

In the NuMI beam approximately $87 \%$ of the incident $120 \mathrm{GeV}$ protons interact in the target, with the majority of the remaining $13 \%$ interacting $715 \mathrm{~m}$ downstream in the hadron absorber [10]. We consider HNL production occurring in both the target and the absorber, the latter giving access to shorter $N$ lifetimes as a result of being significantly closer to the detector. We simulate the particle propagation using GEANT4 [23] and the $\tau^{ \pm}$production using PYTHIA8 [24]. Approximately $10 \%$ of the beam protons reach the absorber with energy $T_{p} \approx 120 \mathrm{GeV}$. For $120 \mathrm{GeV}$ protons interacting in either the target or the absorber, an average of $2.1 \times 10^{-7}\left(3.0 \times 10^{-7}\right) \tau^{+}\left(\tau^{-}\right)$are produced per proton [25]. To generate a flux of $N$, we simulate the decays $\tau^{ \pm} \rightarrow N X$, where $X$ consists of SM particles. We simulate the kinematics by assuming $m_{X}=m_{\pi^{ \pm}}$and that the branching ratio of this new decay is $\operatorname{Br}\left(\tau^{ \pm} \rightarrow N X^{ \pm}\right)=0.9\left|U_{\tau N}\right|^{2} K\left(m_{N}\right)[22,26]$. Since the $D_{(s)}^{ \pm}$ and $\tau^{ \pm}$lifetimes are small, the kinematics of $N$ produced in the target and absorber are qualitatively the same. However, the geometric acceptance of ArgoNeuT is significantly larger for the absorber-produced $N$ due to the proximity to the detector.

The HNL decay products are then simulated in the ArgoNeuT detector using the LArSoft software framework [27], which simulates the particle propagation using GEANT4 [23] then performs detector response simulation and reconstruction [11,28]. A stand-alone version of the MINOS simulation and reconstruction is then used to simulate the tracks exiting ArgoNeuT and entering the MINOS-ND.

Signature.-The HNL decay $N \rightarrow \nu \mu^{+} \mu^{-}$is seen in ArgoNeuT as a pair of minimally ionizing particles (MIPs) that can be matched to a pair of oppositely charged particles in the MINOS-ND. These muons are energetic and highly forward going: with average energy $\left\langle E_{\mu^{ \pm}}\right\rangle \sim 7 \mathrm{GeV}$; average angle with respect to the beam

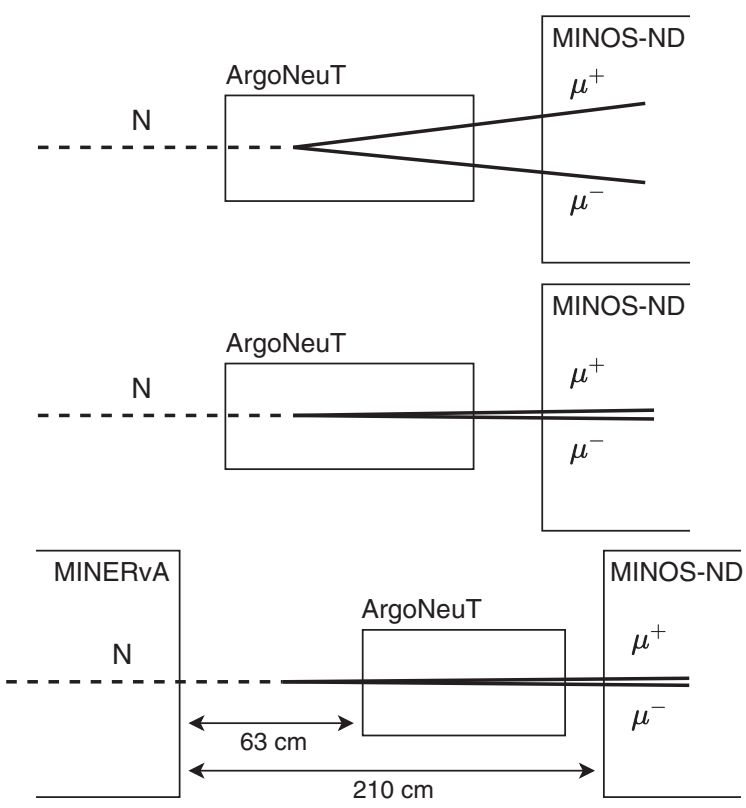

FIG. 1. Diagrams of HNL decays occurring inside the ArgoNeuT detector with the two-track (top) and double-MIP (middle) decay signatures, and of a decay occurring in the cavern between ArgoNeuT and MINERvA with the double-MIP signature (bottom). Diagrams not to scale.

direction $\left\langle\theta_{\text {beam }}\right\rangle \sim 1.5^{\circ}$; and an average opening angle $\left\langle\theta_{\text {opening }}\right\rangle \sim 3^{\circ}$. Given the ArgoNeuT angular resolution of approximately $3^{\circ}$ [29], this results in the muon pair frequently overlapping and being reconstructed as a single track for part or all of their length. Two in-ArgoNeuT decay signatures are therefore considered, each of which is illustrated in Fig. 1 (top, middle). In the first, the muons are reconstructed as two distinct MIP tracks originating from a common vertex, each of which can be matched to tracks in the MINOS-ND. This signature will be referred to as a two-track event. In the second, the muons overlap and are reconstructed as a single track with double-MIP $d E / d x$ for part or all of their length. Then, in the MINOS-ND, the pair of oppositely charged muons separate due to the presence of a magnetic field. This signature will be referred to as a double-MIP event.

In addition to decays occurring inside the ArgoNeuT detector, we also consider decays occurring in the cavern upstream of ArgoNeuT along the NuMI beam line where the resulting muons then pass through the detector. This scenario is illustrated in Fig. 1 (bottom). During the ArgoNeuT physics run, the MINERvA detector [30] was under construction in the upstream cavern. We therefore only consider decays that occur in the $63 \mathrm{~cm}$ between the end of the MINERvA detector and the start of the ArgoNeuT TPC. In this scenario, only the double-MIP signature is considered. This is because the two-track signature is more difficult to distinguish from neutrinoinduced background muons due to the absence of vertex 

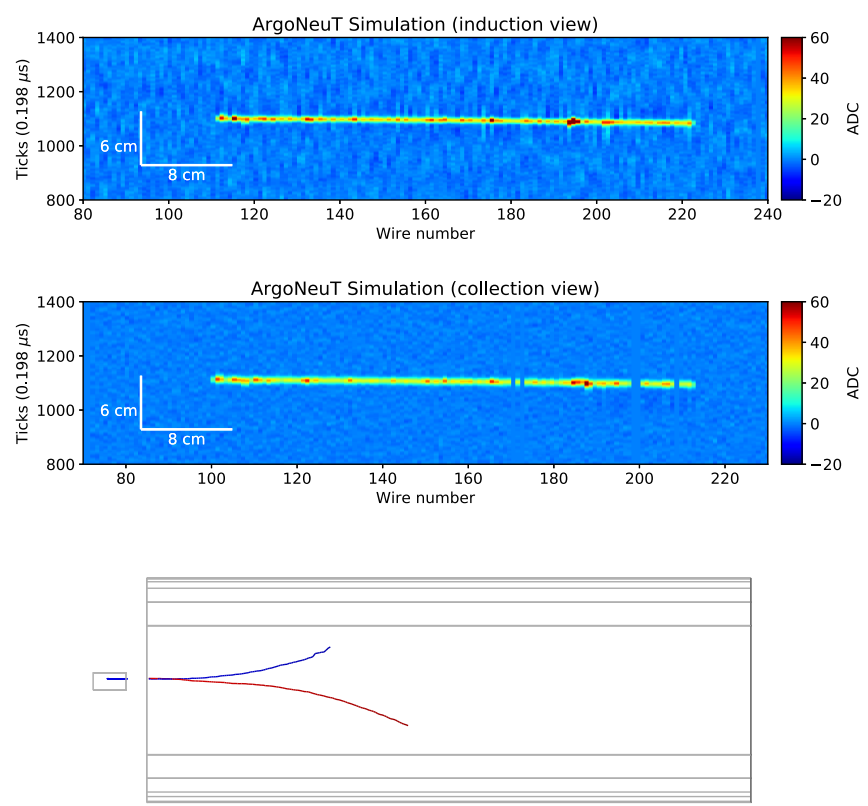

FIG. 2. Event display of a simulated HNL decay with a doubleMIP signature. The induction (top) and collection (middle) wireplane views are shown in ArgoNeuT, where a single track is reconstructed. The color is proportional to the charge deposited. The single track is matched to a pair of tracks in the MINOS-ND (bottom), that are reconstructed with opposite charges represented by the different colors.

information, whereas the double-MIP signature is unique to potential HNL decays.

An example simulated HNL decay with a double-MIP signature is shown in Fig. 2. The pair of muon tracks fully overlap in ArgoNeuT (top, middle), then split once reaching the MINOS-ND (bottom) due to the magnetic field. The strongest identifier of whether a pair of overlapping muons are present is provided by the $d E / d x$ of the track. The region of interest is the start of each track, prior to the muon pair possibly splitting. Figure 3 shows the average reconstructed $d E / d x$ over the first $5 \mathrm{~cm}$ of tracks resulting from simulated HNL decays. Two distinct peaks are visible. The first is at $d E / d x \sim 2 \mathrm{MeV} / \mathrm{cm}$, approximately the $d E / d x$ of a single minimally ionizing muon. For these events the opening angle of the muons is sufficiently large to properly reconstruct them as two separate tracks. The second peak is at $d E / d x \sim 4.5 \mathrm{MeV} / \mathrm{cm}$, approximately double the single MIP $d E / d x$, indicating two overlapping muons. A threshold is applied between the two peaks, illustrated by the dashed line, separating the double-MIP-like and MIP-like populations.

Selection.-A series of preselection cuts are first applied to remove poorly reconstructed events along with obvious non-HNL interactions. The highly forward-going muons from HNL decays can be challenging to reconstruct correctly in LArTPC detectors. This is because the ionisation tracks are near parallel to the readout planes and hence the drifted ionisation charge arrives on the wires at

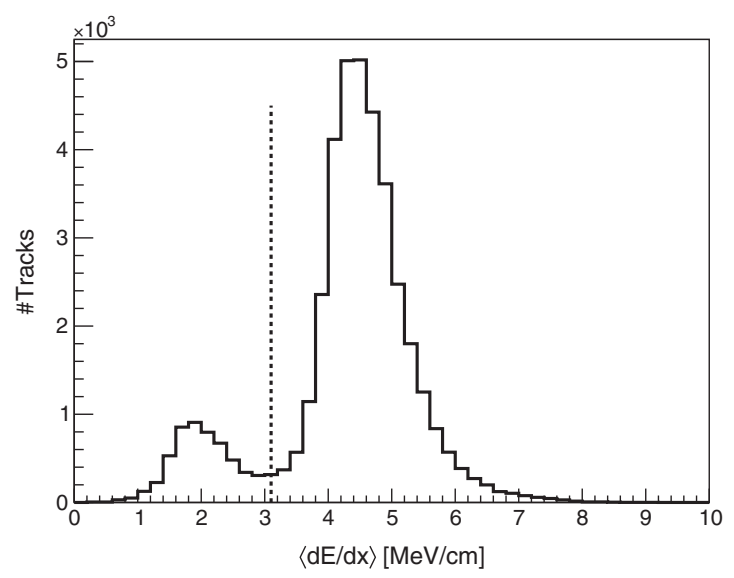

FIG. 3. Average reconstructed $d E / d x$ over the first $5 \mathrm{~cm}$ of tracks resulting from simulated HNL decays. Two peaks are visible, one corresponding to single muons and the other corresponding to two overlapping muons. The threshold applied at $3.1 \mathrm{MeV} / \mathrm{cm}$ is illustrated by a dashed line.

approximately the same time. These events may have large regions missed during the reconstruction and cannot be reliably identified. Therefore, we first remove events with fewer than $80 \%$ of reconstructed energy depositions associated with reconstructed tracks. Next, events with more than three total tracks with length $L \geq 5 \mathrm{~cm}$, or more than two tracks originating from a vertex are removed. This removes any events that are obvious non-HNL interactions due to having additional reconstructed particles present. A harsher cut requiring only two tracks to be present is not applied because a common failure mode of the reconstruction is the presence of split tracks in the region where the overlapping muons begin to separate. Tracks shorter than $L=5 \mathrm{~cm}$ are not considered to avoid removing events that have short $\delta$ rays originating from the muons. Events passing the preselection are then assessed against the two-track and double-MIP selection criteria sequentially.

In the two-track scenario only tracks starting within a fiducial volume in ArgoNeuT are considered, defined as $1 \leq x \leq 46 \mathrm{~cm}$ (drift), $-19 \leq y \leq 19 \mathrm{~cm}$ (vertical), and $z \geq 3 \mathrm{~cm}$ (beam direction), to remove backgrounds originating from the cavern. Events with two tracks that either originate from or can be projected back to a common vertex within the fiducial volume are selected. The tracks are required to be forward going with respect to the beam direction, have length $L \geq 5 \mathrm{~cm}$, exit ArgoNeuT towards the MINOS-ND, have a mean $d E / d x$ over their full length consistent with being a single MIP $(d E / d x<$ $3.1 \mathrm{MeV} / \mathrm{cm}$ ) and have an opening angle between them of $\theta_{\text {opening }} \leq 10^{\circ}$.

In the double-MIP scenario, HNL decays occurring both inside ArgoNeuT and in the upstream cavern are considered. Any events containing tracks with an angle with respect to the beam direction $\theta_{\text {beam }}>15^{\circ}$ are removed, as 
these likely originate from background interactions. The average $d E / d x$ is then calculated over the first 10 hits $(\sim 5 \mathrm{~cm})$ of each track, where any individual anomalously large hits $(d E / d x>10 \mathrm{MeV} / \mathrm{cm})$ are discarded. A cut is applied at $d E / d x>3.1 \mathrm{MeV} / \mathrm{cm}$, illustrated by the dashed line in Fig. 3, to identify events with a possible pair of overlapping muons.

Once candidate events are identified with either the twotrack or double-MIP signature in ArgoNeuT, MINOS-ND matching is performed. Each track is projected to the start of the MINOS-ND and the radial and angular off-sets between the projected tracks and each reconstructed MINOS-ND track are compared. In the two-track case, ArgoNeuT-MINOS-ND matching tolerances of $r_{\text {diff }} \leq$ $12.0 \mathrm{~cm}$ and $\theta_{\text {diff }} \leq 0.17 \mathrm{rad}$ are used [28]. In the double-MIP case, since a single track is being matched to two tracks in the MINOS-ND, the matching tolerances are loosened to 2.5 times the two-track case. The matched tracks are required to be forward going with respect to the beam direction, start within $20 \mathrm{~cm}$ of the up-stream face of the detector and within the calorimeter region, and be at least $1 \mathrm{~m}$ long. This helps to remove any tracks that are unlikely to have originated from ArgoNeuT.

Finally, several selection cuts are applied in the MINOSND. These cuts are the same for both the two-track and double-MIP scenarios. We require that the tracks have an average $d E / d x$ consistent with being a muon (4 $\leq d E / d x \leq 18 \mathrm{MeV} / \mathrm{cm}$ ), are reconstructed with opposite charges, and have start times, $t_{0}$, consistent with having originated from the same interaction or decay: $\left|\Delta t_{0}\right| \leq 20$ ns. Pairs of track with larger $\Delta t_{0}$ could not have originated from a single HNL decay and instead are likely neutrino-induced background muons.

The selection efficiency as a function of the HNL energy, $E_{N}$, is shown in Fig. 4 for simulated $m_{N}=450 \mathrm{MeV} \mathrm{HNL}$ decays occurring inside the ArgoNeuT detector and at two

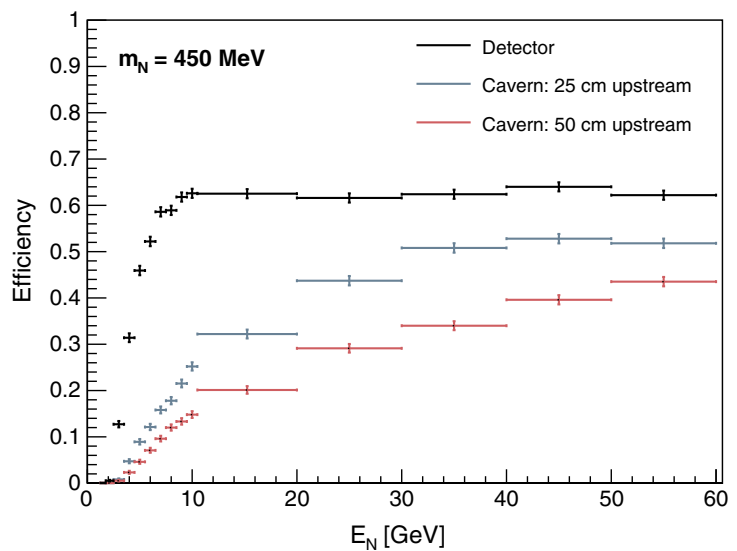

FIG. 4. Selection efficiency as a function of $E_{N}$ for $m_{N}=$ $450 \mathrm{MeV}$ HNL decays occurring inside the ArgoNeuT detector (black) and at 25 (blue) and $50 \mathrm{~cm}$ (red) into the cavern upstream of ArgoNeuT along the beam direction. positions in the upstream cavern. The efficiency inside the detector, defined as the fraction of events that are selected with either the two-track or double-MIP signatures, is around $60 \%-65 \%$ and relatively flat above $E_{N} \sim 10 \mathrm{GeV}$. However, it drops significantly at lower energies predominantly due to one or both of the muons being too low energy to reach the MINOS-ND. The cavern efficiencies are defined as the fraction of decays resulting in muons intersecting with the ArgoNeuT detector that are selected with the double-MIP signature. The further away from ArgoNeuT the decay occurs, the less likely the muon pair is to remain overlapping. This probability decreases further at lower energies where the muons are less forward going.

Backgrounds and systematic uncertainties.-The primary backgrounds in this search originate from misreconstructed neutrino interactions occurring within the ArgoNeuT cryostat, and from neutrino-induced throughgoing muons arising from interactions upstream of the detector. Simulation of these backgrounds is performed with the GENIE [31] neutrino event generator using NuMI beam fluxes provided by the MINERvA Collaboration [32], along with a data-driven model of neutrino-induced through-going muons $[29,33,34]$. In the two track scenario, the dominant form of observed background events are charged current $\nu_{\mu}$ interactions where either the interaction vertex or one or more tracks have been poorly reconstructed leading to these events not being removed. We expect to see $0.1 \pm 0.1$ events of this type in the data. In the double-MIP scenario, the dominant type of background events are single reconstructed muons that have either low energy $\delta$ rays or low energy protons near the track vertex causing them to have a double-MIP-like $d E / d x$. These can then be incorrectly matched to a pair of muons in the MINOS-ND if a second background muon is passing near the ArgoNeuT detector at approximately the same time. We expect to see $0.3 \pm 0.2$ events of this type in the data. The total expected background is therefore $0.4 \pm 0.2$ events.

The systematic uncertainties affecting the measurement are summarized in Table I. They are dominated by the uncertainty on the HNL flux. There is a $20 \%$ uncertainty on the $D_{(s)}^{ \pm}$production [35-37]. Then, the uncertainty on the branching ratios $D_{(s)}^{ \pm} \rightarrow \tau^{ \pm}+\nu_{\tau}$ [38] leads to an additional $5.7 \%$ uncertainty on the $\tau^{ \pm}$flux. Combining these in

TABLE I. Systematic uncertainty impact on the sensitivity.

\begin{tabular}{lc}
\hline \hline Systematic uncertainty & Impact $(\%)$ \\
\hline HNL flux & 20.8 \\
Reconstruction effects & 0.5 \\
Selection efficiency & 3.3 \\
Instrumented volume & 2.2 \\
POT counting & 1.0 \\
Total & 21.2 \\
\hline \hline
\end{tabular}


quadrature leads to a $20.8 \%$ uncertainty on the resulting HNL flux. Next, we consider the impact of uncertainties in the reconstruction by repeating the analysis with each parameter varied individually according to its assigned uncertainty. We apply uncertainties of $3 \%$ on the tuning of the calorimetry [28], 3\% on the track angular reconstruction [29], and $6 \%$ on the energy reconstruction of stopping particles in the MINOS-ND [39]. Finally, we assign a $1 \%$ uncertainty on the charge reconstruction due to the modeling of the magnetic field [39]. Combining the impact of the performed variations in quadrature leads to a $0.5 \%$ systematic uncertainty due to reconstruction effects. In addition to the reconstruction uncertainties, a $3.3 \%$ systematic uncertainty is assigned to the selection efficiency to account for the potential impact of neutrino-induced through-going muons present in $3.3 \%$ of triggers $[11,40]$. A through-going muon registered in coincidence with a HNL event would lead to it being discarded in the preselection. Finally, there is a $2.2 \%$ uncertainty in the size of the ArgoNeuT instrumented volume originating from uncertainty in the electron drift velocity [29] and a $1 \%$ uncertainty in the number of collected POT [34].

Results.-The selection has been applied to the full ArgoNeuT $1.25 \times 10^{20}$ POT antineutrino mode dataset. In total zero events pass, consistent with the expected background rate of $0.4 \pm 0.2$ events. Figure 5 shows our exclusion of parameter space at $90 \%$ confidence level with $1.25 \times 10^{20} \mathrm{POT}$ at ArgoNeuT, assuming production from $\tau^{ \pm}$decays. The limit is evaluated using a Bayesian approach with a uniform prior [41]. The $\pm 1 \sigma$ uncertainty on the expected constraint includes both the uncertainty on the background expectation and the $21.2 \%$ systematic uncertainty on the signal production, combined conservatively. The existing limits from CHARM [42] and DELPHI

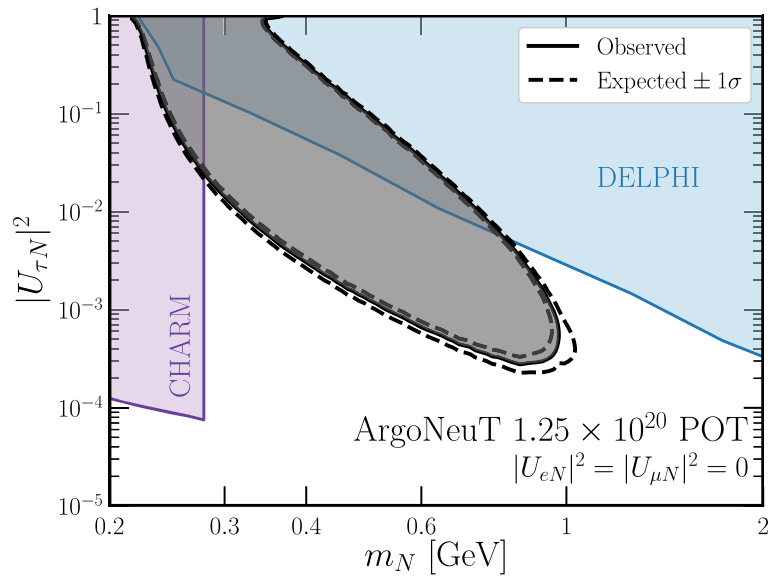

FIG. 5. Constraints on the parameter space at 90\% C.L. from $1.25 \times 10^{20}$ POT at ArgoNeuT (solid black), assuming production from $\tau^{ \pm}$decays. The dashed black lines show the uncertainty on the expected constraint at $\pm 1 \sigma$. The existing limits from CHARM [42] and DELPHI [43] are also shown in purple and blue, respectively.
[43] are also shown in purple and blue, respectively. Our result leads to a significant increase in the exclusion region on the mixing angle $\left|U_{\tau N}\right|^{2}$ of tau-coupled Dirac HNLs with masses $m_{N}=280-970 \mathrm{MeV}$, assuming $\left|U_{e N}\right|^{2}=\left|U_{\mu N}\right|^{2}=0$. Other scenarios are considered in the Supplemental Material [44].

Conclusions.-We have presented the first search for HNLs decaying with the signature $N \rightarrow \nu \mu^{+} \mu^{-}$in a LArTPC detector. Applying a novel technique to identify pairs of overlapping highly forward-going muons, we have searched for tau-coupled Dirac HNLs produced in the NuMI beam and decaying in the ArgoNeuT detector or in the upstream cavern. In the data, corresponding to an exposure to $1.25 \times 10^{20} \mathrm{POT}$, zero passing events are observed consistent with the expected background. The results of this search lead to a significant increase in the exclusion region on the mixing angle $\left|U_{\tau N}\right|^{2}$ of tau-coupled Dirac HNLs with masses $m_{N}=280-970 \mathrm{MeV}$, assuming $\left|U_{e N}\right|^{2}=\left|U_{\mu N}\right|^{2}=0$. The analysis techniques we developed could be applied in future HNL searches performed in larger mass LArTPC experiments.

This manuscript has been authored by Fermi Research Alliance, LLC under Contract No. DE-AC02-07CH11359 with the U.S. Department of Energy, Office of Science, Office of High Energy Physics. We gratefully acknowledge the cooperation of the MINOS Collaboration in providing their data for use in this analysis. We wish to acknowledge the support of Fermilab, the Department of Energy, and the National Science Foundation in ArgoNeuT's construction, operation, and data analysis. This project has received funding from the Science and Technology Facilities Council (STFC), part of the United Kingdom Research and Innovation; and from the Royal Society UK Grants No. RGFIEAl180209 and No. UF140089. We thank Dr. Leigh Whitehead for his assistance processing the MINOS-ND data.

*patrick.green-3@postgrad.manchester.ac.uk

[1] P. Minkowski, Phys. Lett. 67B, 421 (1977).

[2] M. Gell-Mann, P. Ramond, and R. Slansky, Conf. Proc. C 790927, 315 (1979), https://inspirehep.net/literature/9686.

[3] T. Yanagida, Conf. Proc. C 7902131, 95 (1979), https:// inspirehep.net/literature/143150.

[4] S. L. Glashow, NATO Sci. Ser. B 61, 687 (1980).

[5] R. N. Mohapatra and G. Senjanovic, Phys. Rev. Lett. 44, 912 (1980).

[6] J. Schechter and J. W. F. Valle, Phys. Rev. D 22, 2227 (1980).

[7] R. Foot, H. Lew, X. G. He, and G. C. Joshi, Z. Phys. C 44, 441 (1989).

[8] S. Davidson, E. Nardi, and Y. Nir, Phys. Rep. 466, 105 (2008).

[9] A. Boyarsky, M. Drewes, T. Lasserre, S. Mertens, and O. Ruchayskiy, Prog. Part. Nucl. Phys. 104, 1 (2019).

[10] P. Adamson et al., Nucl. Instrum. Methods Phys. Res., Sect. A 806, 279 (2016). 
[11] C. Anderson et al. (ArgoNeuT Collaboration), J. Instrum. 7, P10019 (2012).

[12] D. G. Michael et al. (MINOS Collaboration), Nucl. Instrum. Methods Phys. Res., Sect. A 596, 190 (2008).

[13] F. Bergsma et al. (CHARM Collaboration), Phys. Lett. 166B, 473 (1986).

[14] G. Bernardi et al., Phys. Lett. 166B, 479 (1986).

[15] G. Bernardi et al., Phys. Lett. B 203, 332 (1988).

[16] A. Vaitaitis, R. B. Drucker, J. Formaggio, S. Koutsoliotas, T. Adams et al. (NuTeV and E815 Collaborations), Phys. Rev. Lett. 83, 4943 (1999).

[17] A. V. Artamonov et al. (E949 Collaboration), Phys. Rev. D 79, 092004 (2009).

[18] K. Abe et al. (T2K Collabortaion), Phys. Rev. D 100, 052006 (2019).

[19] P. Abratenko et al. (MicroBooNE Collaboration), Phys. Rev. D 101, 052001 (2020).

[20] E. Cortina Gil et al. (NA62 Collaboration), Phys. Lett. B 807, 135599 (2020).

[21] E. Cortina Gil et al. (NA62 Collaboration), Phys. Lett. B 816, 136259 (2021).

[22] D. Gorbunov and M. Shaposhnikov, J. High Energy Phys. 10 (2007) 015; 11 (2013) 101(E).

[23] S. Agostinelli et al., Nucl. Instrum. Methods Phys. Res., Sect. A 506, 250 (2003).

[24] T. Sjöstrand, S. Ask, J. R. Christiansen, R. Corke, N. Desai, P. Ilten, S. Mrenna, S. Prestel, C. O. Rasmussen, and P.Z. Skands, Comput. Phys. Commun. 191, 159 (2015).

[25] P. Coloma, E. Fernández-Martínez, M. González-López, J. Hernández-García, and Z. Pavlovic, Eur. Phys. J. C 81, 78 (2021).

[26] $K\left(m_{N}\right)<1$ enforces that this vanishes as $m_{N}$ grows larger than $\sim \mathrm{GeV}$. The factor of 0.9 accounts for the $\sim 10 \%$ of $\tau^{ \pm}$ decays into heavier final states that would not allow for the production of a HNL in our mass range of interest.

[27] E. Snider and G. Petrillo, J. Phys. Conf. Ser. 898, 042057 (2017).

[28] R. Acciarri, C. Adams, J. Asaadi, B. Baller, T. Bolton et al. (ArgoNeuT Collaboration), Phys. Rev. D 98, 052002 (2018).
[29] J. Spitz, Ph.D. Thesis, FERMILAB-THESIS-2011-36, Yale University, 2011, https://doi.org/10.2172/1249481.

[30] L. Aliaga et al. (MINERvA Collaboration), Nucl. Instrum. Methods Phys. Res., Sect. A 743, 130 (2014).

[31] C. Andreopoulos et al., Nucl. Instrum. Methods Phys. Res., Sect. A 614, 87 (2010).

[32] L. Aliaga et al. (MINERvA Collaboration), Phys. Rev. D 94, 092005 (2016).

[33] C. Anderson, M. Antonello, B. Baller, T. Bolton, C. Bromberg et al. (ArgoNeuT Collaboration), Phys. Rev. Lett. 108, 161802 (2012).

[34] R. Acciarri, C. Adams, J. Asaadi, B. Baller, T. Bolton et al. (ArgoNeuT Collaboration), Phys. Rev. D 89, 112003 (2014).

[35] S. Barlag et al. (ACCMOR Collaboration), Z. Phys. C 39, 451 (1988).

[36] G. A. Alves, S. Amato, J. C. Anjos, J. A. Appel, J. Astorga et al. (Fermilab E769 Collaboration), Phys. Rev. Lett. 77, 2388 (1996); 81, 1537(E) (1998).

[37] C. Lourenco and H. K. Wohri, Phys. Rep. 433, 127 (2006).

[38] P. Zyla et al. (Particle Data Group), Prog. Theor. Exp. Phys. 2020, 083C01 (2020).

[39] P. Adamson et al. (MINOS Collaboration), Phys. Rev. D 81, 072002 (2010).

[40] C. Anderson et al. (ArgoNeuT Collaboration), J. Instrum. 7, P10020 (2012).

[41] O. Helene, Nucl. Instrum. Methods Phys. Res. 212, 319 (1983).

[42] J. Orloff, A. N. Rozanov, and C. Santoni, Phys. Lett. B 550, 8 (2002).

[43] P. Abreu et al. (DELPHI Collaboration), Z. Phys. C 74, 57 (1997); 75, 580(E) (1997).

[44] See Supplemental Material at http://link.aps.org/ supplemental/10.1103/PhysRevLett.127.121801 for evaluation of the exclusion regions ArgoNeuT can apply to the mixing of electron- and muon-coupled heavy neutral leptons, which includes Ref. [45].

[45] A. Aduszkiewicz et al. (NA61/SHINE Collaborations), Phys. Rev. D 100, 112001 (2019). 